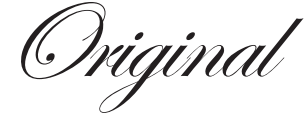

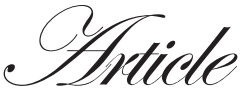

\section{The role of image guided percutaneous drainage in multidisciplinary management of necrotizing pancreatitis}

\author{
Rashesh Solanki, Venumadhav Thumma, R.A.Sastry, N.Bheerappa
}

\section{ABSTRACT}

Department of Surgical

Gastroenterology,

Nizam's Institute of Medical

Sciences,

Punjagutta, Hyderabad . 500082.

India

Correspondence:

Dr. Rashesh Solanki,

Email: raseshsolanki@yahoo.co.in
Background: Percutaneous catheter drainage (PCD) has become popular as a minimally invasive technique in the treatment armamentarium of patients with necrotizing pancreatitis requiring intervention. It obviates surgery and its attendant morbidity in a considerable number of patients in this setting.

Aim: The aim of the study was to examine whether PCD upfront avoids surgery in a cohort of patients with necrotizing pancreatitis requiring intervention, and to identify factors predicting its failure.

Methods: We retrospectively reviewed demographic, clinical, and perioperative details of patients with severe acute pancreatitis from January 2009 to December 2011. Of 40 patients with necrotizing pancreatitis admitted to the surgical gastroenterology unit at our institute, 23 patients requiring PCD or surgical intervention were included in this study. Patients with successful and failed PCD were compared to identify factors predictive of failure of PCD. We also compared patients undergoing open necrosectomy with those undergoing PCD upfront, and open necrosectomy in terms of major complications including death.

Results:. Surgery was avoided in 7/23 (30\%) patients with necrotizing pancreatitis who underwent PCD upfront. Higher APACHE II score $(\mathrm{p}=0.003)$ and extent of intrapancreatic necrosis $(>50 \%, \mathrm{p}=0.03$ ) were statistically significant predictors of failure of PCD. Although the complications were not different in patients undergoing PCD upfront followed by surgery, they had lower APACHE II and SOFA scores after PCD and had resolution of organ failures. Conclusions: PCD obviates surgery or acts as a temporizing measure in a significant number of patients with necrotizing pancreatitis. APACHE II scores and extent of intrapancreatic necrosis are principle factors determining success of PCD.

KEYWORDS: necrotizing pancreatitis, percutaneous catheter drainage, severe acute pancreatitis, APACHE II score, CT severity index.

\section{Introduction}

Necrotizing pancreatitis, which develops in about $20 \%$ of patients with acute pancreatitis, is associated with a death rate of 8 to $39 \%^{1}$. Although sterile pancreatic necrosis can be managed expectantly, infected pancreatic necrosis virtually always requires intervention, traditionally with open necrosectomy ${ }^{2}$. This approach, however, is associated with a profound physiological insult to an already critically ill patient, leading to mortality rates of around $25-35 \%$. Thus, there is a need to minimise the magnitude of intervention and therefore several minimally invasive techniques like percutaneous 
catheter drainage(PCD), video-assisted retroperitoneal debridement(VARD) ${ }^{4}$ and endoscopic necrosectomy ${ }^{5}$ have become popular. Furthermore, PCD has been shown to obviate the need of surgery in a recent randomised controlled study ${ }^{4}$. The present study was performed to evaluate the role of PCD in multidisciplinary management of necrotizing pancreatitis and identify factors associated with failure of PCD.

\section{Material and methods}

This was a retrospective study from a prospectively collected data cluster, from January 2009 to December 2011. Of the 40 patients with necrotizing pancreatitis admitted to the surgical gastroenterology unit at Nizam's institute of Medical Sciences, a tertiary referral hospital in South India,23 patients with necrotizing pancreatitis who required intervention were included in the study.

The therapy for all patients with necrotizing pancreatitis (study population) included goal directed fluid resuscitation, parenteral antibiotics (usually a Carbapenem), enteral nutrition and organ support when required. The indication for intervention was infected pancreatic necrosis ${ }^{6}$ identified by gas within the necrosis or pancreatic necrosis with clinically evident sepsis not responding to antimicrobial therapy. Variables recorded included demographic data, clinical profile, etiology, APACHE II scores ${ }^{7}$ at admission and 24 hours after surgery, organ failure, at admission and postoperatively, sequential organ failure assessment(SOFA) $\operatorname{scores}^{8}$, at admission and postoperatively, radiological findings including CT severity index (CTSI $)^{9}$ operative and procedural details, ICU admission and stay, and complications including death. The study was approved by the institutional Ethics Committee and all subjects consented to the study.

Organ failures were classified according to Atlanta criteria ${ }^{10}$. In addition, CNS failure was also taken in account. Thus the seven organ failures were defined as : shock: Systolic blood pressure $<90 \mathrm{mmHg}$; respiratory failure: $\mathrm{PaO} 2 \leq 60 \mathrm{mmHg}$; renal failure: serum creatinine: $\geq 177 \mu \mathrm{mol} / 1$ or $\geq 2 \mathrm{mg} / \mathrm{dl}$ after rehydration; gastrointestinal bleeding: $500 \mathrm{ml}$ in $24 \mathrm{~h}$; disseminated intravascular coagulation: platelets $\leq 100,000 / \mathrm{mm}^{3}$, fibrinogen $<1.0 \mathrm{~g} / 1$ and fibrin-split products $>80 \mu \mathrm{g} / \mathrm{l}$; severe metabolic disturbances: calcium $\leq 1.87 \mathrm{mmol} / 1$ or $\leq 7.5 \mathrm{mg} / \mathrm{dl}$ and central nervous system failure: if the Glasgow coma score was $<13$. Persistent organ failure was defined as organ failure persisting beyond 48 hours $^{11}$.
These patients were divided into percutaneous catheter drainage (PCD) alone, surgical drainage alone and PCD followed by surgical drainage. Patients who were successfully treated with percutaneous drainage $(n=7)$, were compared with those who failed the same $(n=7)$ to identify predictive factors of failure of percutaneous drainage. Those who underwent percutaneous drainage followed by surgery $(n=6)$ were compared with those who underwent surgery upfront $(n=9)$.

PCD was performed in patients where a liquid component of the necrosum was identified on ultrasound and CECT abdomen and adequate access was obtained. It was not limited to patients with 'walled-off' necrosis. In patients with predominant solid necrosis, an initial surgery was considered. In general, the clinical status of the patient did not influence the choice of the procedure, although critically ill patients on mechanical ventilation were not subjected to PCD.

\section{Percutaneous drainage}

Under ultrasound guidance, $12-20 \mathrm{~F}$ pigtail drains were placed transgastrically or retroperitoneally into the necrosum. Following PCD, the drains were flushed with sterile saline twice daily. They were manipulated in the event of reduction in output or blockage. Additional percutaneous intervention was performed if feasible in patients with ongoing clinical sepsis. In patients recovering from sepsis the drains were removed if the output became minimal and ultrasound abdomen did not demonstrate a residual collection and the output was not amylase rich. Patients with amylase rich drain or persistent purulent drain with no clinical sepsis were discharged and followed up on outpatient basis every 2 weeks until the drain output reduced, when the drains were removed.

Failure of percutaneous drainage was defined as persisting clinical sepsis with absence of drainable collections as demonstrated on ultrasound examination of abdomen or requirement of surgery to control sepsis.

\section{Surgery}

The extent of surgery was guided by CT as a 'road map'. The lesser sac was entered through gastrocolic omentum or through the mesocolon. Necrosectomy was done by minimum blunt dissection and suction. Pairs of large drains were placed in the lesser sac and continuous irrigation initiated in select cases; for instance in patients with early surgery and inadequate 
debridement at index surgery.Intraoperative bacterial and fungal culture of the necrosum and/or pus was sent.

\section{Statistical analysis}

Continuous variables were expressed as mean \pm SD (standard deviation) and categorical variables as number (percentage). Continuous variables were analyzed with t-test and continuous with chi-square or Fisher's exact test as appropriate. The analysis was performed with SPSS software version 15.

\section{Results}

The baseline demographic and clinical profile of the study population who required intervention is given in Table 1. Out of 23 patients with pancreatic necrosis who underwent intervention, 20 had proven infected necrosis, 3 had sterile necrosis. 8 were treated with percutaneous drainage alone (6 infected necrosis, 2 sterile necrosis), 6 underwent percutaneous drainage followed by surgery (all had infected necrosis) and 9 patients underwent surgery only ( 8 infected necrosis and 1 sterile)(Table 2). The average duration from onset of symptoms to intervention was 33 days. The duration of indwelling catheter varied from 1 to 4 weeks, with an average duration of 13 days.

Overall, systemic inflammatory response syndrome(SIRS) ${ }^{12}$ was present in 22(95.6\%) patients, and organ failure was present in $7(30.4 \%)$, of which $4(17.4 \%)$ were persistent. 4 patients $(17.4 \%)$ required preoperative ICU admission. Mean APACHE II score and SOFA score were $8.83 \pm 4.22$ and $1.82 \pm 1.82$, respectively. $4(17.39 \%)$ patients died. The mean number of interventions in the percutaneous group was 1.5.

Surgery was avoided in 7/23 (30\%) patients with pancreatic necrosis who underwent PCD upfront. On comparing patients who underwent percutaneous drainage successfully $(n=7)$ with those that failed the same $(n=7)$, higher APACHE II score (6.86 \pm 3.53 versus $11.89 \pm 3.37$ respectively, $\mathrm{p}=0.003)$, and extent of necrosis ( $>50 \%$ intrapancreatic necrosis, $\mathrm{p}=0.03$ ) were statistically significant, whereas CTSI $(p=0.072)$ was approaching significance.(Table 3) (Figures 1 and 2). Extent of intrapancreatic necrosis was treated as a continous variable $(<30 \%, 30-50 \%$ and $>50 \%)$. One patient who underwent PCD and whose sepsis was temporarily under control, was discharged, but succumbed later to septic shock as he could not be admitted to our unit due to logistic reasons.

Surgery alone $(n=9)$ and percutaneous drainage followed by surgery $(n=6)$ were comparable in terms of demographic
Table 1: Demographic and clinical profile of study population

\begin{tabular}{lccc}
\hline & $\begin{array}{c}\text { Number of } \\
\text { patients } \\
(\mathbf{n}=\mathbf{2 3})\end{array}$ & $\begin{array}{c}\text { Primary } \\
\text { PCD* } \\
(\mathbf{n = 1 4})\end{array}$ & $\begin{array}{c}\text { Primary } \\
\text { surgery } \\
(\mathbf{n = 0 9})\end{array}$ \\
\hline Age & $38.13 \pm 12.37$ & $35.85 \pm 12.50$ & $41.67 \pm 11.98$ \\
Male : Female & $22: 1$ & $14: 0$ & $8: 1$ \\
SIRS & 22 & 13 & 09 \\
Organ failures at admission & 07 & 05 & 02 \\
Multi organ failures & 03 & 01 & 02 \\
Persistent organ failures & 04 & 02 & 02 \\
Pre operative ICU & 04 & 02 & 02 \\
admission & & & \\
APACHE II score at & $8.83 \pm 4.22$ & $6.92 \pm 3.45$ & $11.89 \pm 3.37$ \\
admission & & & \\
SOFA scores at & $1.82 \pm 1.82$ & $1.5 \pm 1.55$ & $2.33 \pm 2.18$ \\
admission & & & \\
Extent of necrosis & & 07 & 02 \\
- <30\% & 09 & 02 & 00 \\
- 30-50\% & 02 & 05 & 07 \\
- >50\% & 12 & $7.71 \pm 2.39$ & $8.75 \pm 2.38$ \\
CT severity index & $8 \pm 2.37$ &
\end{tabular}

Continuous variables are expressed as mean \pm SD and categorical as number

*includes patients $(n=6)$ that underwent subsequent surgery due to failed PCD

Table 2 : Type of intervention and necrosis in study population.

\begin{tabular}{lccc}
\hline $\begin{array}{l}\text { Type of } \\
\text { intervention }\end{array}$ & $\begin{array}{c}\text { Number of } \\
\text { patients } \\
(\mathbf{n = 2 3})\end{array}$ & $\begin{array}{c}\text { Infected } \\
\text { necrosis } \\
(\mathbf{n = 2 0})\end{array}$ & $\begin{array}{c}\text { Sterile } \\
\text { necrosis } \\
(\mathbf{n = 3})\end{array}$ \\
\hline Percutaneous drainage & 8 & 6 & 2 \\
Surgery only & 9 & 8 & 1 \\
$\begin{array}{l}\text { Percutaneous drainage } \\
\text { followed by surgery }\end{array}$ & 6 & 6 & 0 \\
\hline
\end{tabular}

Table 3: Comparison of successful and failed percutaneous drainage

\begin{tabular}{lccc}
\hline Study variables & $\begin{array}{c}\text { Successful } \\
(\mathbf{n}=7)\end{array}$ & $\begin{array}{c}\text { Failed } \\
(\mathbf{n}=7)\end{array}$ & P value \\
\hline Age in years & $35.85 \pm 12.51$ & $41.67 \pm 11.98$ & 0.282 \\
Gender (M;F) & $7: 0$ & $7: 0$ & 1.000 \\
Pre-op ICU stay & $1(14.3 \%)$ & $1(14.3 \%)$ & 1.000 \\
SIRS & $7(100.0 \%)$ & $7(100.0 \%)$ & 1.000 \\
Organ failure at admission & $1(14.3 \%)$ & $4(57.1 \%)$ & 0.266 \\
Pre-op SOFA & $1.50 \pm 1.56$ & $2.33 \pm 2.19$ & 0.296 \\
APACHE at admission & $6.86 \pm 3.53$ & $11.89 \pm 3.37$ & 0.003 \\
Extent of necrosis & & & \\
- <30\% & $5(71.4 \%)$ & $2(28.6 \%)$ & 0.286 \\
- 30-50\% & $2(28.6 \%)$ & 0 & 0.462 \\
- >50\% & 0 & $5(71.4 \%)$ & 0.021 \\
Extra pancreatic necrosis & $4(57.1 \%)$ & $6(85.7 \%)$ & 0.559 \\
CT severity index* & $6.67 \pm 2.57$ & $8.85 \pm 1.68$ & 0.072 \\
Infected Necrosis & $5(71.4 \%)$ & $7(100.0 \%)$ & 0.462 \\
\hline
\end{tabular}

$\overline{\text { Continuous variables are expressed as mean }+S D \text { and categorical as }}$ number.

*Median(inter-quartile range) 


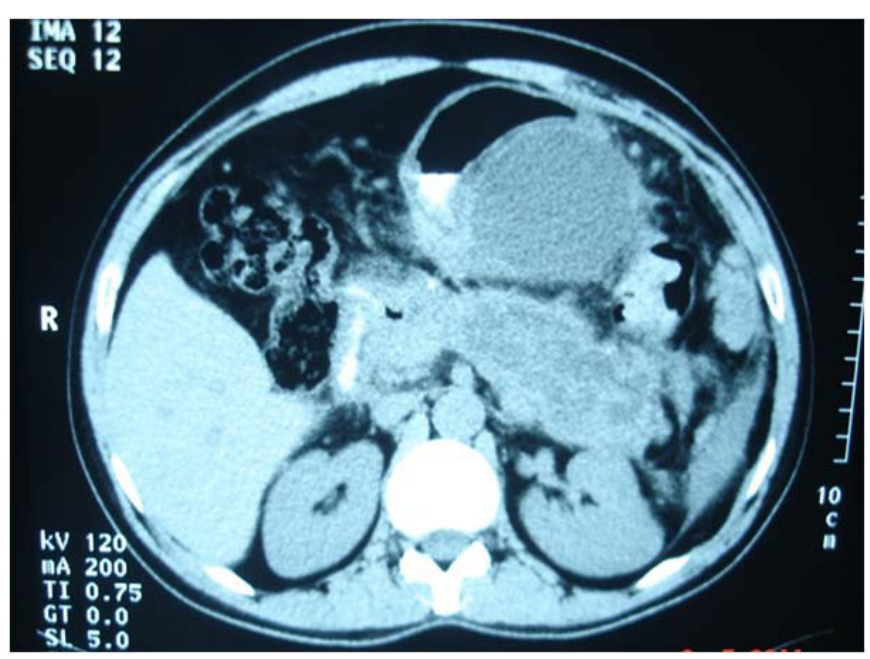

Figure 1: Infected necrosis in body and tail region - successfully treated with PCD

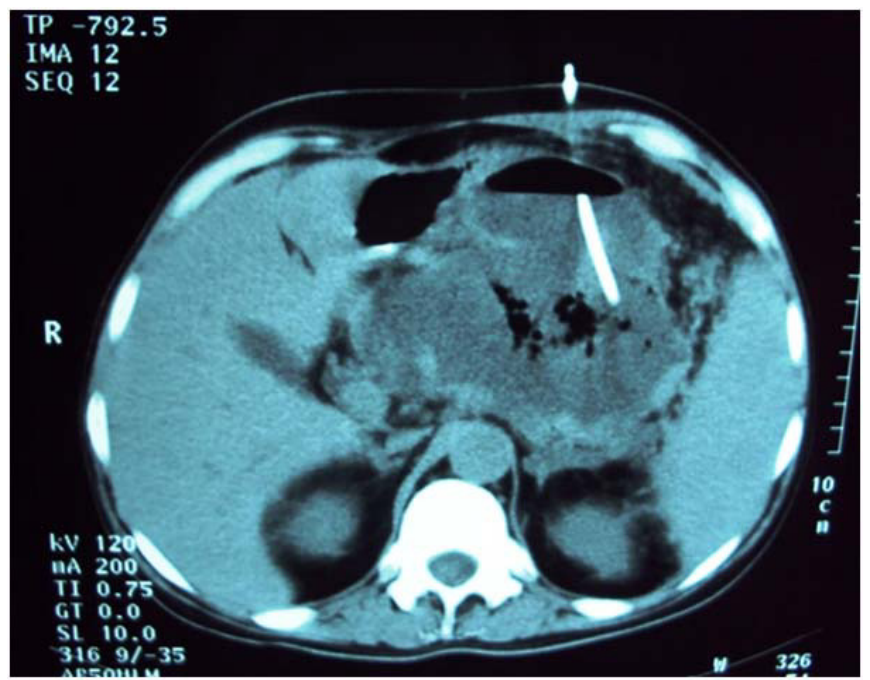

Figure 2: Extensive intrapancreatic $(>50 \%)-$ failed PCD

data, preoperative variables, APACHE II scores at admission and SOFA scores and extent of necrosis. On comparing these groups, preoperative APACHE II scores $(6.33 \pm 2.25$ versus $11.44 \pm 4.25$ respectively, $\mathrm{p}=0.019$ ), were lower in the percutaneous drainage followed by surgery group than surgery alone group. Two patients were discharged with drain in-situ but were readmitted and operated on for recurrent sepsis. The postoperative complications, like new onset organ failures/ ICU admission, systemic and intra-abdominal complications and postoperative stay and mortality were not statistically significant between the two groups.

Complications of PCD were external pancreatic fistula defined as catheter fluid amylase three times the upper normal limit of serum value ${ }^{13}$ which occurred in 5 out of 14 patients(35\%), recurrent fluid collections after catheter removal in 3 out of 14 patients( $21 \%$ ), self limiting bleeding in 1 patient and death in 1 patient. The complications from intervention are shown in Table 4.
Table 5 : PCD as a "temporizing measure"

\begin{tabular}{lcc}
\hline Parameter & Before PCD & After PCD \\
\hline APACHE (mean) & 8.5 & 5.5 \\
SOFA & 2.5 & 1.33 \\
Organ failure & $2 / 6$ & $0 / 6$ \\
\hline
\end{tabular}

The remaining 17 patients with necrotizing pancreatitis did not require intervention and were managed with supportive therapy and antibiotics to control sepsis. This group also included 2 patients with early severe acute pancreatitis and multi-organ failure who succumbed even with maximum supportive therapy.

\section{Discussion}

Image guided percutaneous catheter drainage (PCD) upfront avoids surgery in a significant proportion of patients with necrotizing pancreatitis ${ }^{4,14}$. In the landmark PANTER trial ${ }^{4}, 35 \%$ of patients avoided surgery only by percutaneous drainage. In the present study, in about $30 \%$ patients, surgery was obviated. In a systematic review from the same group, a pooled analysis of eleven studies revealed that surgery was obviated in as many as $214(55.7 \%)$ of 384 patients $^{14}$.

Furthermore, PCD temporizes critically ill patients to faciliatate later interevention in a less sick condition ${ }^{14-16}$ which is suggested by the observation that in 6 patients who underwent percutaneous drainage before surgery, two who had organ failures had reversal of the same and mean APACHE II and SOFA scores were lower after percutaneous drainage (8.5 versus 5.5 and 2.5 versus 1.33 respectively) (Table 5).

Out of the 20 patients who had infected pancreatic necrosis, 6 were managed with PCD alone and antimicrobial therapy. Conventionally, surgery is mandatory in this group of patients ${ }^{17}$. We, therefore, hypothesize that a select group of patients with infected pancreatic necrosis can be managed non-

Table 4: Complications in the intervention group

\begin{tabular}{lccc}
\hline $\begin{array}{l}\text { Post-intervention } \\
\text { complications (n=23) }\end{array}$ & $\begin{array}{c}\text { Post- } \\
\text { operative }\end{array}$ & $\begin{array}{c}\text { Post- } \\
\text { percutaneous } \\
\text { drainage }\end{array}$ & Total \\
\hline Pancreatic fistula & 4 & 5 & 9 \\
Recurrent pancreatitis & 1 & 0 & 1 \\
Pseudocyst/fluid collections & 4 & $3^{\#}$ & 6 \\
Upper GI bleeding & 2 & 0 & 2 \\
Intra-abdominal bleed* & 2 & 1 & 3 \\
Colonic fistula & 1 & 0 & 1 \\
Biliary stricture & 1 & 0 & 1 \\
Death & 3 & 1 & 4 \\
\hline
\end{tabular}

\# in patients who underwent PCD alone

*one patient had both upper GI bleeding and intra-abdominal bleeding from inferior pancreatoduodenal archade aneurysm which was successfully angio-embolised. 
surgically by a combination of image-guided percutaneous drainage and antimicrobial therapy. Hence, it is important to identify this subset of patients.

It has been suggested that central gland necrosis responds poorly to $\mathrm{PCD}^{16}$. In a study by Freeny etal ${ }^{18}$, of 34 patients with infected pancreatic necrosis, 16(47\%) avoided surgery. Of these, 12 had necrosis of body and tail, whereas only 4(29\%) out of 14 patients who had central gland managed toavoid surgery. Accordingly, in our study, extensive necrosis (more than 50\% intra-pancreatic) was associated with failure of PCD. This observation stands to reason as pancreatic parenchymal necrosis is more likely to be solid and less amenable to percutaneous drainage alone.

The success of PCD in necrotizing pancreatitis is technique dependent. More aggressive management with mechanical removal of solid necrotic element by upsizing drains and sequentially dilating the $\operatorname{tract}^{15}$, snare and Dormia baskets ${ }^{19,20}$ or placement of multiple catheters, facilitates a more complete removal of necrosis and ensures a lower failure rate. We did not employ any of these techniques, although in a few patients we did place multiple catheters and performed repeated procedures as required, provided they were feasible. Nevertheless, we feel our study is applicable to a less resource intensive setting as well.

We also compared patients undergoing PCD followed by surgery $(n=6)$, with those undergoing surgery $(n=9)$, to assess whether PCD upfront reduced the complication rate as was demonstrated in the PANTER trial, although in the latter study, PCD was followed if required by video-assisted retroperitoneal debridement (VARD). Since the complication rates were not different between the groups, we may conclude that it provides an indirect evidence that once an open necrosectomy is performed, it inflicts considerable physiological trauma and leads to higher complication rates as compared to minimally invasive approaches.

Alternatively, PCD is a useful adjunct in management of postoperative residual or recurrent fluid collections ${ }^{21} .3$ patients in our study required PCD following necrosectomy with one of them requiring it twice.

There are inherent limitations in our study. First, the numbers in each group are low. Second, patients in the successful PCD group had lower APACHE II scores than those who failed PCD, suggesting that there was a selection bias involved and the latter group was sicker than the former.

In summary, percutaneous drainage upfront avoids surgery in a considerable number of selected patients with necrotizing pancreatitis. Patients who have a large amount of intrapancreatic necrosis, (more than 50\%) are more likely to require further procedures to achieve complete debridement. Percutaneous drainage upfront in patients requiring surgery was a temporizing measure in our study and did not reduce postoperative complications.

\section{References}

1. Banks PA, Freeman ML. Parameters Committee of the American College of Gastroenterology. Practice guidelines in acute pancreatitis. Am J Gastroenterol. 2006;101:2379-2400.

2. Working Party of the British Society of Gastroenterology; Association of Surgeons of Great Britain and Ireland; Pancreatic Society of Great Britain and Ireland;Association of Upper GI Surgeons of Great Britain and Ireland. UK guidelines for the management of acute pancreatitis. Gut. 2005;54:iii1-9.

3. Connor S, Ghaneh P, Raraty M, Rosso E, Hartley MN, Garvey $\mathrm{C}$, et al. Increasing age and APACHE II scores are the main determinants of outcome from pancreatic necrosectomy. $\mathrm{Br} J$ Surg. 2003;90:1542-8.

4. van Santvoort HC, Besselink MG, Bakker OJ, Hofker HS, Boermeester MA, Dejong CH, et al. A step-up approach or open necrosectomy for necrotizing pancreatitis. $N$ Engl J Med. 2010;362:1491-502.

5. Baron TH, Thaggard WG Morgan DE, Stanley RJ. Endoscopic therapy for organized pancreatic necrosis. Gastroenterology. 1996;3:755-64.

6. Uhl W, Warshaw A, Imrie C, Bassi C, McKay CJ, Lankisch PG, et al. IAP Guidelines for the Surgical Management of Acute Pancreatitis. Pancreatology. 2002;2:565-73.

7. Larvin M, McMahon MJ. APACHE-II score for assessment and monitoring of acute pancreatitis. Lancet. 1989;2:201-5.

8. Vincent JL, de Mendonça A, Cantraine F, Moreno R, Takala J, Suter PM, et al. Use of the SOFA score to assess the incidence of organ dysfunction/failure in intensive care units: results of a multicenter, prospective study. Working group on "sepsis-related problems" of the European Society of Intensive Care Medicine. Crit Care Med. 1998;26:1793-1800.

9. Balthazar EJ. Acute pancreatitis:assessment of severity with clinical and CT evaluation. Radiology. 2011;223:603-13.

10. Bradley EL III. A clinically based classification system for acute pancreatitis. Summary of the International Symposium on Acute Pancreatitis, Atlanta, Ga, September 11 through 13, 1992. Arch Surg. 1993;128:586-90.

11. UK guidelines for the management of acute pancreatitis. Working Party of the British Society of Gastroenterology; Association of Surgeons of Great Britain and Ireland; Pancreatic Society of Great Britain and Ireland; Association of Upper GI Surgeons of Great Britain and Ireland. Gut. 2005;54:iii1-9.

12. Bone RC, Balk RA, Cerra FB, Dellinger RP, Fein AM, Knaus WA, et al. Definitions for sepsis and organ failure and guidelines for the use of innovative therapies in sepsis. The ACCP/SCCM Consensus Conference Committee. American College of Chest 
Physicians/Society of Critical Care Medicine. 1992. Chest. 2009; 136:e28.

13. Bassi C, Dervenis C, Butturini G, Fingerhut A, Yeo C, Izbicki J, et al. Postoperative pancreatic fistula: an international study group (ISGPF) definition. Surgery. 2005;138:8-13.

14. van Baal MC, van Santvoort HC, Bollen TL, Bakker OJ, Besselink MG, Gooszen HG; Dutch Pancreatitis Study Group. .Systematic review of percutaneous catheter drainage as primary treatment for necrotizing pancreatitis. $\mathrm{Br} J$ Surg. 2011;98:18-27.

15. Windsor JA. Infected pancreatic necrosis : drain first but do it better. HPB (Oxford). 2011;13:367-8.

16. Navaneethan U, Vege SS, Chari ST, Baron TH. . Minimally invasive techniques in pancreatic necrosis. Pancreas. 2009;38:867-75.

17. Büchler MW, Gloor B, Müller CA, Friess H, Seiler CA, Uhl W.
Acute necrotizing pancreatitis: treatment strategy according to the status of infection. Ann Surg. 2000;232:619-26.

18. Freeny PC, Hauptmann E, Althaus SJ, Traverso LW, Sinanan M. Percutaneous CT-guided catheter drainage of infected acute necrotizing pancreatitis: techniques and results. AJR Am J Roentgenol. 1998;170:969-75.

19. Echenique AM, Sleeman D, Yrizarry J, Scagnelli T, Guerra JJ Jr, Casillas VJ, et al. Percutaneous catheter directed debridement of infected pancreatic necrosis: results in 20 patients. $J$ Vasc Interv Radiol. 1998;9:565-71.

20. Mann S, Gmeinwieser J, Schmidt J,Zirngibl H, Jauch KW. Possibilities and borderlines of interventional therapy in necrotizing pancreatitis. Zentralbl Chir. 2001;126:15-22.

21. Mamlouk MD, vanSonnenberg E. Interventional Radiology for Necrotizing pancreatitis. J Gastrointest Surg. 2011;15:1101-3. 